\title{
Variabilité génétique de quinze provenances roumaines d'épicéa commun (Picea abies (L) Karst). Premiers résultats *
}

\author{
B Héois **, H Van de Sype \\ INRA, station d'amélioration des arbres forestiers, Ardon 45160 Olivet, France
}

(Reçu le 22 mars 1990; accepté le 27 décembre 990)

\begin{abstract}
Résumé - Quinze provenances roumaines d'épicéa commun d'origines naturelle et artificielle, structurées en 118 familles issues de pollinisation libre, sont testées sur 2 sites (Gennes, près de Besançon et Amance, près de Nancy). Deux provenances françaises, Gérardmer et Chapois, et un polycross sont utilisées comme témoins. Les caractères étudiés sont la hauteur à 10 ans, le débourrement végétatif, l'angle de branchaison et la fourchaison.

Comparées aux témoins, les populations roumaines sont vigoureuses, assez tardives (sauf les 2 provenances artificielles) et ont un bon angle de branchaison. La fourchaison présente une corrélation défavorable avec la hauteur $\left(r=0,55^{*}\right)$ et l'angle de branchaison $\left(r=0,57^{*}\right)$. L'utilisation des provenances CIMP et MOLD permettrait, par rapport aux meilleurs témoins, un gain de $27 \%$ sur la hauteur et de $13 \%$ sur l'angle de branchaison avec un débourrement presqu'aussi tardif que Chapois, témoin de tardiveté.

L'étude des paramètres génétiques montre une absence de variabilité interfamiliale pour 2 provenances. D'après l'homogénéité des variances-covariances, les autres provenances sont regroupées en 2 ensembles sur Gennes (dont l'un est composé des 2 provenances artificielles) et en un seul sur Amance. Les héritabilités au sens strict sont de 0,16-0,37 pour l'angle de branchaison et de $0,32-$ 0,46 pour la hauteur; celles du débourrement sont un peu supérieures $(0,24-0,78)$. Les corrélations génétiques additives sont en général favorables mais non significatives.

Les gains génétiques espérés par intercroisement des 50 meilleurs individus sélectionnés sur un index combiné individu-famille sont, par rapport aux meilleurs témoins, de $34 \%$ sur la hauteur et de $17 \%$ sur l'angle de branchaison, avec un débourrement aussi tardif que Chapois.

Ces résultats montrent lintérêt de quelques provenances roumaines et particulièrement de certaines origines de Bucovine et du Bihor. lls devront être confirmés à un âge moins juvénile et pour d'autres caractères.
\end{abstract}

Plcea ables / Roumanie / provenance / descendance / hauteur / débourrement / angle de branchalson / fourchaison / héritabllité au sens strict / corrélation génétique

Summary - Genetic variability of fifteen Romanian provenances of Nonway spruce (Picea ables (L) Karst) : preliminary results. A collection of 15 Romanian provenances of Norway spruce has been studied: 13 are natural and originate from Bucovine (north-eastern), Bihor (north-western) and central Romania; 2 others are artificial. They are represented by 118 open-pollinated progenies

\footnotetext{
* Cet article fait suite à un travail de DEA (génétique et amélioration des plantes, INAPG) réalisé par B Héois sous la conduite de H Van de Sype

** Correspondance et tirés à part
} 
and compared with 3 standards: 2 French provenances and a polycross. The material was sown in 1979 and planted in 1982 at 2 sites located in the Eastern part of France (Gennes, near Besançon; and Amance, near Nancy). The traits studied here are the height at $10 \mathrm{yr}$, bud burst in 1986, branch angle and forkness. Compared to the standards, the Romanian provenances are fast-growing, relatively late flushing (except the 2 artificial ones) and have a good branch angle. The provenance effect for this last trait seems to be weaker than for growth or flushing (table III). Forkness (table $V$ ) has an unfavourable correlation with total height $\left(r=0.55^{*}\right)$ and branch angle $\left(r=0.577^{*}\right)$. The provenance $x$ site interaction for height is significant, but caused by one unstable provenance only (fig 3). Two stable provenances may be selected: 1 from Bucovine and 1 from Bihor. Compared to the best standard, they give a gain of $27 \%$ for total height, $13 \%$ for branch angle, and with almost the same flushing time as the latest standard (table VI). The study of genetic parameters shows a lack of variability between families for 2 provenances originating from the central area. According to the variancescovariances homogeneity, the other provenances are pooled into 2 groups in Gennes (one is composed of the 2 artificial provenances) and only 1 group in Amance. Narrow sense heritability (table VII) is $0.16-0.37$ for branch angle, 0.32-0.46 for height, and is a little higher for flushing (0.24-0.78). Genetic additive correlations between traits are generally favourable but not significant (table VIII). Forkness has an unfavourable correlation with total height $\left(0.25^{* *}\right)$ and branch angle $\left(0.36^{* *}\right)$, as at the provenance level. The family $x$ site interaction is only significant for height, but is caused by only one unstable family. The expected genetic gains for a clonal seed orchard option are estimated by a multi-trait combined individual and family selection and compared to the best standards, the $50 \mathrm{hi}$ gher index give an expected genetic gain of $34 \%$ for $10 \mathrm{yr}$ height and $17 \%$ for branch angle and are as late as the Chapois standard (table IX). These results show the interest of some Romanian provenances particularly those from Bucovine and Bihor, but this should be confirmed at a less juvenile stage and for other traits.

Picea abies / Romania / provenance / progeny / height / bud burst / branch angle / forkness / narrow sense heritablilty/genetic correlation

\section{INTRODUCTION}

La stratégie d'amélioration génétique de l'épicéa commun menée par l'INRA s'est déroulée en 2 étapes. La première a été l'exploration de sa variabilité au sein des 3 grandes régions géographiques de son aire naturelle (décrite par Schmidt-Vogt, 1977): zones alpine, hercyniennecarpatique et nordique. Cette variabilité a été étudiée en particulier dans une expérience IUFRO comportant 1100 provenances. Sur l'unique dispositif français, les résultats préliminaires montraient l'intérêt de la zone hercynienne-carpatique (Ferrand, 1986) pour nos conditions de reboisement, et particulièrement de certaines populations de Roumanie. Ainsi 4 d'entre elles se plaçaient parmi les meilleures (pour un critère «débourrement + hauteur à 17 ans"). Cependant une partie de la variabilité observée demeurait inexpliquée : 9 provenances originaires des mêmes régions et situées aux mêmes altitudes que les meilleures présentaient des performances médiocres).

La deuxième étape est l'étude des paramètres génétiques des meilleures populations dans le but de les intégrer, le cas échéant, dans la population d'amélioration de l'espèce. En 1976, à la demande de I'INRA, V Enescu, améliorateur forestier Roumain, procurait des descendances issues de pollinisation libre (“open») et récoltées dans 15 provenances roumaines. Le test mis en place avec ce matériel est, à notre connaissance, le seul en Europe de l'Ouest rassemblant des descendances originaires de cette partie de l'aire naturelle. II est maintenant possible d'en tirer 
les premières informations, de préciser les performances et d'estimer les paramètres génétiques de ces populations.

\section{MATÉRIEL ET MÉTHODES}

\section{Matériel}

Le matériel végétal est constitué de 118 descendances «openn récoltées dans 15 provenances roumaines. Une provenance regroupe un ensemble d'arbres susceptibles de s'interpolliniser et ayant des caractéristiques génétiques communes. Treize provenances sont naturelles et proviennent de 3 régions montagneuses, la $\mathrm{Bu}$ covine, le Centre et le Bihor (fig 1, tableau I).
Deux autres sont artificielles et appartiennent à la variété Europaea (Enescu, communication personnelle). Cette collection est comparée à 3 témoins, d'une part 2 provenances françaises situées à une altitude de $600 \mathrm{~m}$, l'une naturelle, vigoureuse et précoce, Gérardmer (notée GERA), l'autre artificielle et tardive, Chapois (notée CHAP), et d'autre part, un polycross Gérardmer $x$ Istebna (noté GIST), Istebna étant une provenance vigoureuse des Beskides polonaises.

Le matériel a été semé au printemps 1979 puis repiqué en pépinière un an plus tard. En 1982, il a été planté sur les sites de Gennes (près de Besançon) et d'Amance (près de Nancy), en racines nues, avec des écartements de $2 \mathrm{~m} \times 2 \mathrm{~m}$. Les dispositifs sont en blocs incomplets à parcelle unitaire monoarbre (tableau II).

Tableau I. Caractéristiques des provenances roumaines.

\begin{tabular}{|c|c|c|c|c|c|c|c|c|c|}
\hline Régions & $\operatorname{Cod} \theta$ & Nom de la & \multicolumn{2}{|c|}{ Lat N } & \multicolumn{2}{|c|}{ Long $E$} & $\begin{array}{r}\text { Altit } \\
(m)\end{array}$ & $N b$ & Fam \\
\hline Artificielle (1) & $\begin{array}{l}\text { BEIU } \\
\text { DOBR }\end{array}$ & $\begin{array}{l}\text { Beius } \\
\text { Dobresti }\end{array}$ & $\begin{array}{l}46 \\
46\end{array}$ & $\begin{array}{l}45 \\
44\end{array}$ & $\begin{array}{l}22 \\
22\end{array}$ & $\begin{array}{l}23 \\
23\end{array}$ & $\begin{array}{l}500 \\
500\end{array}$ & $\begin{array}{l}8 \\
9\end{array}$ & $\begin{array}{r}9 \\
10\end{array}$ \\
\hline Bihor (1) & $\begin{array}{l}\text { BELI } \\
\text { TURD } \\
\text { CIMP }\end{array}$ & $\begin{array}{l}\text { Belis } \\
\text { Turda } \\
\text { Cimpeni }\end{array}$ & $\begin{array}{l}46 \\
46 \\
46\end{array}$ & $\begin{array}{l}31 \\
30 \\
25\end{array}$ & $\begin{array}{l}23 \\
23 \\
23\end{array}$ & $\begin{array}{l}01 \\
45 \\
10\end{array}$ & $\begin{array}{l}1200 \\
1200 \\
1250\end{array}$ & $\begin{array}{l}5 \\
7 \\
5\end{array}$ & $\begin{array}{r}8 \\
8 \\
10\end{array}$ \\
\hline Bucovine (2) & $\begin{array}{l}\text { MOLD } \\
\text { MARG } \\
\text { FRAS } \\
\text { POJO } \\
\text { DOCI }\end{array}$ & $\begin{array}{l}\text { Moldovita } \\
\text { Marginea } \\
\text { Frasin } \\
\text { Pojorita } \\
\text { Dorna Cindreni }\end{array}$ & $\begin{array}{l}47 \\
47 \\
47 \\
47 \\
47\end{array}$ & $\begin{array}{l}40 \\
35 \\
28 \\
22 \\
17\end{array}$ & $\begin{array}{l}25 \\
25 \\
25 \\
25 \\
25\end{array}$ & $\begin{array}{l}28 \\
34 \\
48 \\
46 \\
05\end{array}$ & $\begin{array}{r}900 \\
670 \\
750 \\
1000 \\
1000\end{array}$ & $\begin{array}{l}3 \\
3 \\
1\end{array}$ & $\begin{array}{l}5 \\
7 \\
2 \\
6 \\
8\end{array}$ \\
\hline Centre. (3) & $\begin{array}{l}\text { SINM } \\
\text { COMA } \\
\text { NEAS } \\
\text { NEHO }\end{array}$ & $\begin{array}{l}\text { Sin-Martin } \\
\text { Comandau } \\
\text { Nehoias } \\
\text { Nehoiu }\end{array}$ & $\begin{array}{l}46 \\
45 \\
45 \\
45\end{array}$ & $\begin{array}{l}13 \\
45 \\
26 \\
30\end{array}$ & $\begin{array}{l}25 \\
26 \\
26 \\
26\end{array}$ & $\begin{array}{l}57 \\
20 \\
10 \\
30\end{array}$ & $\begin{array}{r}900 \\
1150 \\
1090 \\
1140\end{array}$ & $\begin{array}{l}8 \\
8 \\
9\end{array}$ & $\begin{array}{r}10 \\
8 \\
10 \\
7\end{array}$ \\
\hline Harghita (4) & $\begin{array}{l}\text { GURG } \\
\text { Nombr } \\
\text { Nombr }\end{array}$ & $\begin{array}{l}\text { Gurghiu } \\
\text { tal de provenanc } \\
\text { tal de familles pa }\end{array}$ & 46 & 50 & 24 & 50 & 1200 & $\begin{array}{r}7 \\
13 \\
74\end{array}$ & $\begin{array}{r}10 \\
\\
15 \\
118\end{array}$ \\
\hline
\end{tabular}

Les nombres entre parenthèses renvoient à la figure 1. Le nombre de familles (Nb Fam) est indiqué pour Gennes (G) et Amance (A). 


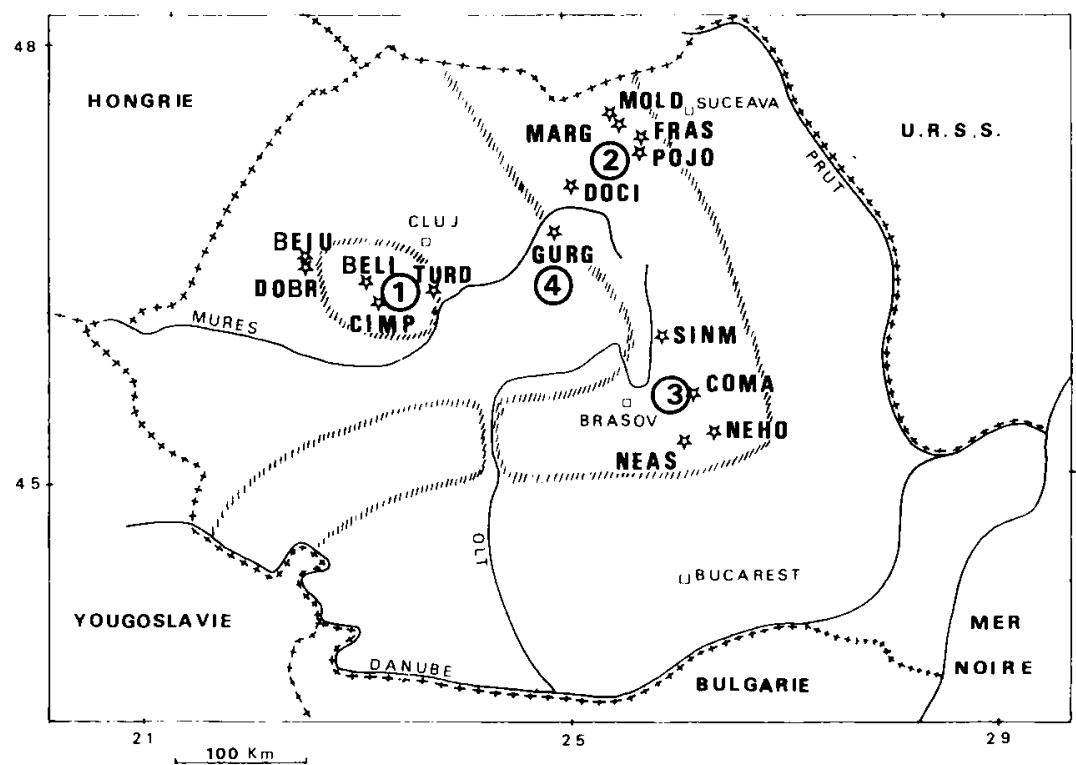

Fig 1. Répartition des provenances roumaines étudiées; Bihor (1), Bucovine (2), Centre (3), Harghita (4).

\section{Caractères mesurés}

La vigueur des arbres a été appréciée par la hauteur mesurée de 6 à 10 ans depuis la graine (notées $\mathrm{H}_{6}, \mathrm{H}_{8}, \mathrm{H}_{10}$ ). La tardiveté du débourrement végétatif (notée $\mathrm{DEB}$ ) a été estimée à 8 ans par la date d'ouverture du bourgeon terminal notée en 1986 à partir d'une date origine (5 mai à Amance, 9 mai à Gennes). L'angle de branchaison par rapport à la verticale (noté ANG) a été mesuré en degrés sur la plus grosse branche du verticille situé à $1,30 \mathrm{~m}$ de hauteur environ.

Tableau II. Localisation et caractéristiques des dispositifs.

Gennes

Franche Comté

Doubs

$430 \mathrm{~m}$

très profond

calcaire

Limono-argileux

Profondeur

Sous-sol

74

73

42
Amance

Lorraine

Meurthe-et-Moselle

$240 \mathrm{~m}$

$0,40-0,80 \mathrm{~m}$

argile compacte

118

40 
La fourchaison (FOUR) a été notée selon une modalité de présence-absence d'une ou plusieurs fourches. D'autres caractéristiques susceptibles d'être associées à ce défaut (Portefaix, 1987) ont été notées selon les mêmes modalités à 7 et 10 ans sur la pousse de l'année : il s'agit de rameaux latéraux (RLAT, RLAT $_{10}$ ) et de pousses d'août terminales (PTER7, PTER ${ }_{10}$ ). L'analyse de ces caractères qualitatifs (présence-absence) n'a pu tenir compte de l'effet bloc.

\section{Méthodes d'analyse des caractères quantitatifs}

Les analyses statistiques sont réalisées à l'aide de la programmathèque Amance qui permet de traiter les dispositits déséquilibrés et non orthogonaux (Bachacou et al, 1981).

L'étude au niveau provenance est réalisée par la décomposition de la variance suivant le modele croisé 1 :

$$
X_{i k n}=\mu+p_{i}+b_{k}+p b_{i k}+E_{i k n}
$$

avec la moyenne générale $(\mu)$, les effets provenance $\left(p_{i}\right)$, bloc $\left(b_{k}\right)$ et d'interaction provenance $x$ bloc $\left(\rho b_{i k}\right)$ considérés comme fixes, et l'erreur résiduelle aléatoire $E_{i k n}$.

Toutes les analyses ultérieures s'effectuent à partir des valeurs ajustées à l'effet bloc.

L'interaction génotype $x$ site est analysée selon le même modèle croisé; les effets principaux sont considérés comme fixes. Si l'interaction est significative, l'instabilité d'un génotype est estimée par son écovalence (Wricke, 1962) rapportée au total des écovalences (écovalence relative, Baradat, 1984).

Les paramètres génétiques de chaque provenance (i) sont estimés selon le modèle 2, l'effet famille $\left(F_{i N}\right)$ étant supposé aléeatoire :

$$
Y_{i j n}=\mu_{i}+F_{j i}+E_{i j n}
$$

Pour augmenter la précision des paramètres génétiques, les provenances sont regroupées en ensembles statistiquement homogènes. La fonction $\chi^{2}$ de Kullback (in: Legendre et Legendre, 1984), permet de tester l'homogénéité des variances-covariances intrafamiliales puis interfamiliales de chaque groupe. Pour ces groupes, l'effet famille $F_{i j i}$ est alors analysé selon le modèle hiérarchique 3, le facteur provenance étant pris comme fixe :

$$
Y_{i j n}=\mu+p_{i}+F_{j i}+E_{i j n}
$$

La variance génétique additive est estimée par 4 fois la variance interfamiliale, sous les hypothèses de populations infinies, de reproduction en panmixie et d'individus non-apparentés. L'espérance de gain génétique additif obtenu par l'intercroisement des meilleurs individus est calculée à partir d'une sélection multicaractère combinée individu-famille réalisée à partir d'un index; celui-ci est une combinaison linéaire des estimations des valeurs génétiques additives des caractères entrant dans l'index (Christophe, 1979). Les intervalles de confiance des héritabilités ont été calculés selon Becker (1984).

Quelques limites de l'étude doivent être soulignées :

- les 15 provenances étudiées ne sont pas représentatives de toute l'aire roumaine de l'épicéa commun; la valeur des provenances est appréciée d'après un nombre très restreint de représentants (tableau I);

- le petit nombre de représentants et l'absence d'information sur les modalités de l'échantillonnage altèrent la valeur et la précision des paramètres génétiques estimés;

- le regroupement de populations qui n'échangent plus de gènes introduit un biais dans l'estimation des espérances de gain.

La signification des tests est symbolisée par : ns (non significatif), " (significatif à $P \leq 5 \%$ ), ** (significatif à $P \leq 1 \%$ ).

\section{RÉSULTATS}

Les pousses annuelles (fig 2) sont croissantes de 1985 à 1988 (sauf en 1987, à Gennes) pour atteindre $0,68 \mathrm{~m}$ à Gennes et $1,05 \mathrm{~m}$ à Amance. Le caractère pousse annuelle n'est donc pas encore stabilisé. Ainsi, la dernière hauteur totale $\left(\mathrm{H}_{10}\right)$ sera utilisée comme seul estimateur de la vigueur. Le débourrement moyen à 8 ans correspond au 30 mai 1986 à Gennes, au 


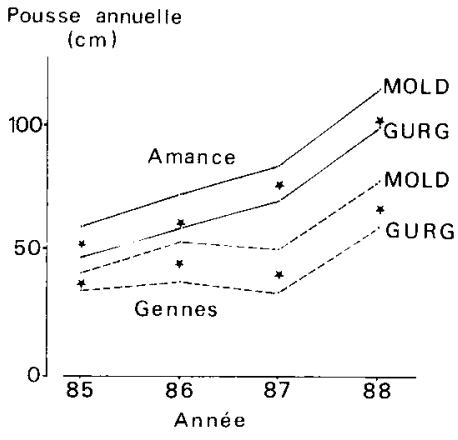

Flg 2. Evolution des pousses annuelles de 2 provenances roumaines et de la moyenne $\left(^{*}\right)$ pour chaque site.

25 mai 1986 à Amance. L'angle moyen de branchaison (ANG) est satisfaisant $\left(67^{\circ}\right.$ à Gennes et $70^{\circ}$ à Amance) mais son coefficient de variation phénotypique totale (10$13 \%$ ) est particulièrement faible comparativement à ceux des autres caractères (19$36 \%$ ). Les corrélations phénotypiques sont favorables entre la vigueur et l'angle de branchaison, et défavorables entre la vigueur et le débourrement.

\section{Niveau provenance}

L'analyse de la variance (modèle 1) montre que quelle que soit la variable considérée, les effets provenance et bloc sont toujours significatifs à $1 \%$ (tableau III). A Amance, les $F$ du facteur provenance pour la hauteur totale augmentent de 8 à 17 de 1984 à 1988, ce qui traduit une discrimination meilleure des provenances au fil des ans.

L'interaction provenance $\times$ bloc n'est significative que sur le site de Gennes et uniquement pour la hauteur des premières années $\left(\mathrm{H}_{6}-\mathrm{H}_{8}\right)$. L'interaction provenance $x$ site n'est significative ni pour le débourrement $\left(F_{15 / 3636}=1,3 \mathrm{~ns}\right)$ ni pour l'angle de branchaison ( $F=1,5 \mathrm{~ns}$ ). Elle l'est en revanche pour $\mathrm{H}_{10}\left(F=4,0^{* *}\right)$ comme le met en évidence la figure 3. Toutefois, l'élimination de $\mathrm{DOCl}$ (écovalence $=36 \%$ ), représentée par une seule descendance, suffit à supprimer l'interaction. En outre, la corrélation entre moyennes de provenance sur les 2 sites est de $0,85^{\star \star}$. Pour ces 3 caractères, les provenances étudiées sont donc globalement stables à la fois aux niveaux bloc et site.

Tableau III. F de Fisher calculés selon le modèle 1.

\begin{tabular}{lccccccc}
\hline $\begin{array}{l}\text { Sources de } \\
\text { variation }\end{array}$ & & $D D L$ & $H_{6}$ & $H_{8}$ & $H_{10}$ & $D E B$ & ANG \\
\hline Provenance & $\mathrm{G}$ & 15 & $24^{* *}$ & $26^{* *}$ & $26^{* *}$ & $27^{* *}$ & $21^{* *}$ \\
Provenance & $\mathrm{A}$ & 17 & $8^{* *}$ & $12^{* *}$ & $17^{* *}$ & $16^{* *}$ & $10^{* *}$ \\
Bloc & $\mathrm{G}$ & 72 & $2,0^{* *}$ & $2,0^{* *}$ & $2,4^{* *}$ & $2,4^{* *}$ & $2,5^{\star *}$ \\
Bloc & $\mathrm{A}$ & 39 & $3,5^{* *}$ & $3,3^{* *}$ & $3,6^{* *}$ & $1,4^{* *}$ & $3,5^{* *}$ \\
Prov x Bloc & $\mathrm{G}$ & 834 & $1,15^{*}$ & $1,12^{*}$ & $1,05 \mathrm{~ns}$ & $0,9 \mathrm{~ns}$ & $0,9 \mathrm{~ns}$ \\
Prov x Bloc & $\mathrm{A}$ & 622 & $1,0 \mathrm{~ns}$ & $0,9 \mathrm{~ns}$ & $0,9 \mathrm{~ns}$ & $1,0 \mathrm{~ns}$ & $0,9 \mathrm{~ns}$ \\
\hline
\end{tabular}

(A pour Amance, G pour Gennes). Les degrés de liberté résiduels sont de 1320 sur Gennes et de 1399 sur Amance. 
Les provenances françaises sont peu vigoureuses (fig 3). La médiocre performance de Gérardmer peut résulter de l'emploi d'un lot de graines peu représentatif de ce massif de 479 ha réputé pour sa vigueur. Le croisement GIST est un peu supérieur à la moyenne. Les provenances roumaines sont généralement vigoureuses. Celles du Bihor se distinguent de celles de la zone Centre par une vigueur supérieure sur les 2 sites. Les performances des autres provenances sont disparates. Les provenances artificielles BEIU et DOBR se situent aux alentours de la moyenne. Parmi les provenances naturelles, 2 de Bucovine (MOLD, FRAS) et une du Bihor (CIMP) se remarquent par leur vigueur. Toutefois, les provenances de Bucovine étudiées à Gennes sont constituées par un nombre très réduit de des-

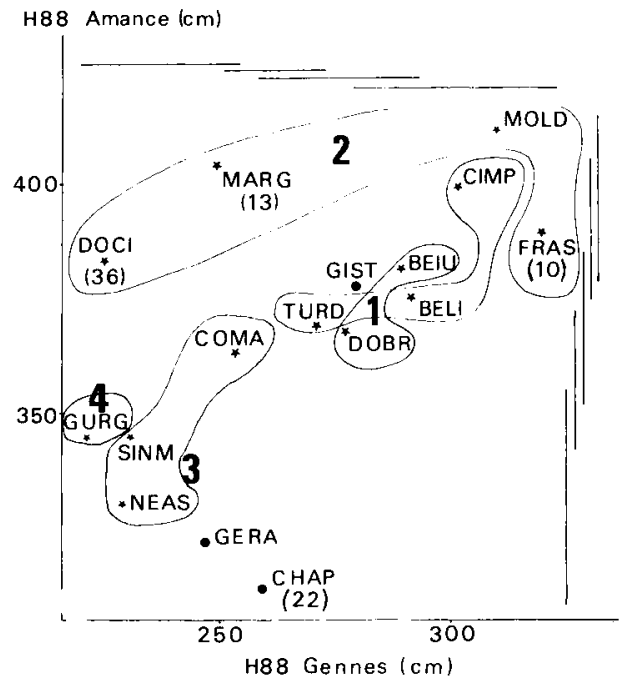

Fig 3. Hauteurs moyennes des provenances sur les 2 sites (seules les descendances communes aux 2 sites sont prises en considération). L'écovalence relative des provenances les plus instables est indiquée entre parenthèses. Les lignes verticales et horizontales regroupent les moyennes qui ne sont pas différentes au seuil de $5 \%$; Bihor (1), Bucovine (2), Centre (3), Harghita (4). cendances (tableau 1). Elles sont, par conséquent, peu représentatives de la valeur de leur peuplement d'origine.

Le débourrement de la provenance Gérardmer est le plus précoce (18 mai 1986 à Amance et 24 mai 1986 à Gennes). La provenance Chapois, témoin de tardiveté, est effectivement classée parmi les plus tardives (28 mai à Amance et 31 mai à Gennes), tout comme le croisement GIST (fig 4). Les provenances roumaines d'origine artificielle sont significativement précoces alors que celles d'origine naturelle, dont CIMP et MOLD, sont tardives.

Pour l'angle de branchaison, le classement des provenances est comparable à celui décrit pour la vigueur : les provenances françaises sont relativement médiocres $\left(61-64^{\circ}\right)$, les provenances naturelles du Bihor et de Bucovine, en particulier CIMP et MOLD, sont parmi les meilleures $\left(72-73^{\circ}\right)$.

La fréquence des défauts est faible, notamment à Gennes et en 1985 (tableau IV); leur étude ne sera donc abordée que sur le site d'Amance. Les écarts phénotypiques entre provenances sont plus impor-

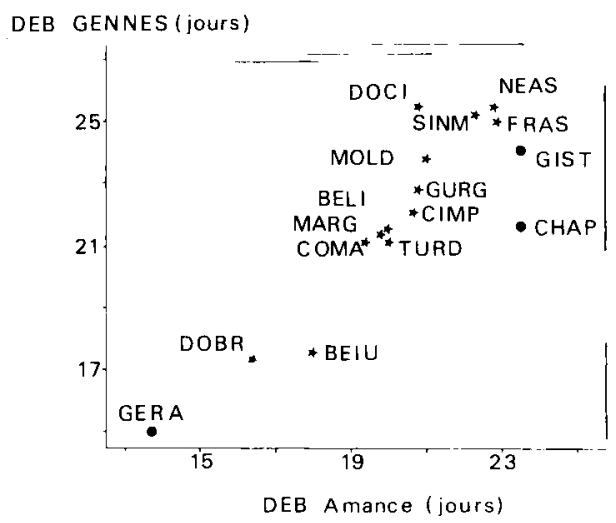

Fig 4. Débourrements moyens des provenances (représentées par les descendances communes) sur les 2 sites. Les lignes verticales et horizontales regroupent les moyennes qui ne sont pas différentes au seuil de $5 \%$. 
Tableau IV. Fréquences (\%) moyennes et extrêmes des caractères qualitatifs au niveau provenance.

Gennes
moy minimaxi moy mini maxi

\begin{tabular}{lrlrr}
\hline RLAT $_{7}$ & 7 & $(4-13)$ & 22 & $(8-36)^{*}$ \\
PTER $_{7}$ & 1 & $(0-6)$ & 1 & $(0-5)^{*}$ \\
RLAT $_{10}$ & 13 & $(4-19) *$ & 35 & $(13-54)^{*}$ \\
PTER $_{10}$ & 2 & $(0-7)$ & 12 & $(0-22)^{*}$ \\
FOUR & 3 & $(0-7) *$ & 12 & $(3-17)^{*}$ \\
\end{tabular}

RLAT : rameaux latéraux sur la pousse de l'année; PTER : pousse d'aodt terminale sur la pousse de l'année; " : les fréquences extrêmes sont significativement différentes au seuil de $5 \%$.

tants pour les caractères de pousse d'août (PTER) et de rameaux latéraux (RLAT) que pour la fourchaison. Toutefois, la présence de pousse d'août et de rameaux latéraux sur terminal n'est pas significativement corrélée à la fourchaison (tableau V). De plus, le caractère PTER n'est pas "stable" dans le temps (corrélation 7-10 ans $=0,25 \mathrm{~ns}$ ). En première analyse, ces 2 caractères ne semblent donc pas apporter de précision sur la prédiction de la fourchaison. La fréquence de ce défaut paraît réduite pour les 2 provenances françaises $(2-8 \%)$ et plus importante pour les provenances roumaines (MOLD: $10 \%$ et CIMP: $18 \%)$.

La corrélation de rang entre les caractères quantitatifs (tableau V) n'est significative qu'entre hauteur et angle de branchaison : elle est positive et favorable. Les caractères qualitatifs semblent associés défavorablement avec la vigueur; la fourchaison l'est également avec l'angle de branchaison.

Dans l'ensemble, les provenances naturelles roumaines sont supérieures aux 2 provenances françaises pour tous les caractères quantitatifs (tableau VI). Par rapport à la meilleure provenance française, les provenances MOLD (Bucovine) et CIMP (Bihor) présentent une supériorité minimale de $17-25 \%$ sur la vigueur et de $13 \%$ sur la branchaison, tout en maintenant une tardiveté aussi bonne que celle de Chapois.

Tableau V. Corrélations de rang entre 6 caractères pour les moyennes des 18 provenances sur Amance.

\begin{tabular}{lccccc}
\hline & RLAT & FOUR & $H_{10}$ & DEB \\
\hline PTER $_{10}$ & $0,91^{* *}$ & & & & \\
FOUR & $0,38 \mathrm{~ns}$ & $0,33 \mathrm{~ns}$ & & & \\
$\mathrm{H}_{10}$ & $0,70^{* *}$ & $0,77^{* *}$ & $0,55^{*}$ & & \\
DEB & $-0,43 \mathrm{~ns}$ & $-0,42 \mathrm{~ns}$ & $-0,10 \mathrm{~ns}$ & $-0,11 \mathrm{~ns}$ & \\
ANG & $0,37 \mathrm{~ns}$ & $0,39 \mathrm{~ns}$ & $0,57^{*}$ & $0,75^{* *}$ & $-0,34 \mathrm{~ns}$ \\
& & & & & \\
\hline
\end{tabular}

RLAT : rameaux latéraux sur la pousse de l'année; PTER : pousse d'août terminale sur la pousse de l'année. 
Tableau VI. Comparaison des performances des 2 meilleures provenances roumaines. (A pour Amance et G pour Gennes).

\begin{tabular}{|c|c|c|c|c|c|c|c|c|}
\hline \multirow[t]{2}{*}{ Prov } & \multicolumn{2}{|c|}{$H_{10}(\mathrm{~cm})$} & \multicolumn{2}{|c|}{$D E B(i)$} & \multicolumn{2}{|c|}{$A N G\left(^{\circ}\right)$} & \multicolumn{2}{|c|}{ FOUR (\%) } \\
\hline & $A$ & $G$ & $A$ & $G$ & $A$ & $G$ & $A$ & G \\
\hline \multicolumn{9}{|c|}{ Moyenne générale (témoins compris) } \\
\hline & 368 & 266 & 20 & 21 & 70 & 67 & 12 & 3 \\
\hline \multicolumn{9}{|l|}{ Témoins } \\
\hline CHAP & 311 & 258 & 24 & 22 & 64 & 61 & 8 & B \\
\hline GERA & 321 & 249 & 14 & 15 & 64 & 63 & 3 & 2 \\
\hline \multicolumn{9}{|c|}{ Meilleures provenances roumaines } \\
\hline MOLD & 414 & 310 & 21 & 24 & 72 & 72 & 11 & 4 \\
\hline CIMP & 400 & 302 & 20 & 22 & 73 & 73 & 18 & 1 \\
\hline
\end{tabular}

\section{N/veau descendance}

L'analyse de la fourchaison a été réalisée sans tenir compte du niveau provenance. Les coefficients de corrélation calculés sur les moyennes de descendances entre fourchaison, vigueur et angle sont inférieurs à ceux observés au niveau provenance mais vont dans le même sens $(r=$ $0,25^{* *}$ entre FOUR et $\mathrm{H}_{10}, \mathrm{r}=0,36^{* *}$ entre FOUR et ANG). La distribution des valeurs de fourchaison (présence-absence) ne permet pas d'utiliser ce caractère dans un index de sélection individu-famille.

L'interaction famille $x$ site n'est significative à $5 \%$ que pour la hauteur $\left(F_{73 / 3636}=\right.$ $\left.1,4^{*}\right)$. Mais l'élimination de la famille DOCl01, seul représentant de la provenance instable DOCl, supprime toute interaction. Par ailleurs, l'interaction famille $x$ année n'est significative ni pour la hauteur totale $\left(F_{3 / 219}=0,2 \mathrm{~ns}\right)$ ni pour la pousse annuelle ( $F=0,6 \mathrm{~ns})$. Le classement des descendances est donc comparable d'une année à l'autre et dans les 2 sites.

L'analyse de la variance réalisée pour chaque provenance (modèle 2), montre que l'effet famille n'est pas significatif dans la moitié des cas. En particulier, les provenances GURG et NEAS, bien qu'ayant un nombre relativement important de descendances (de 7-10), ne présentent aucun effet famille significatif, quel que soit le caractère. Cette absence de variabilité résulte d'une variance interfamiliale faible et non pas d'une variance résiduelle anormalement élevée. Le faible nombre de descendances par provenance incite à les regrouper en ensembles homogènes. Seront exclues les provenances constituées d'un trop petit nombre de familles ou présentant un $F$ de l'effet famille $<1$ pour au moins un caractère. Les estimations sont faites avec toutes les réserves indiquées à la section Matériel et Méthodes.

Pour le site de Gennes, le test de Kullback intrafamilial, pour les caractères $\mathrm{H}_{10}$, DEB et ANG, montre que les provenances ne constituent pas un groupe homogène $\left(\chi^{2}=116,9^{\star *}\right.$ pour $\left.60 \mathrm{dd}\right)$. L'examen des corrélations intrafamiliales permet de définir 2 groupes homogènes de provenances. Le premier groupe (GR1) est constitué des 33 descendances de BELI, CIMP, COMA, SINM, TURD. Le second groupe (artificielles) comprend les 17 provenances arti- 
ficielles BEIU et DOBR, soit 17 descendances. Cette distinction des provenances artificielles paraît valider a posteriori le regroupement opéré. Les provenances de Bucovine n'ont pas été retenues pour les raisons indiquées précédemment.

Pour le site d'Amance, les tests de Kullback intra- et interfamiliaux ne sont pas significatifs. Le groupe GRT composé de 12 provenances, soit 96 descendances, est donc considéré comme homogène. De plus, il nous a semblé intéressant de distinguer sur Amance le sous-groupe des provenances artificielles (BEIU et DOBR) afin de le comparer sur les 2 sites. $\mathrm{Ce}$ sous-groupe de 19 descendances est statistiquement homogène.

\section{Paramètres génétiques et sélection}

Pour les 3 caractères, et quel que soit le groupe considéré, la répartition de la variance d'après le modèle 3 est quasiment identique : $10 \%$ pour la variance interfamiliale, $90 \%$ pour la variance résiduelle. Dans l'ensemble, les héritabilités de la hauteur et de l'angle de branchaison ne sont pas significativement différentes les unes des autres ainsi qu'entre les groupes (tableau VII). L'héritabilité du débourre- ment est très élevée pour le groupe des provenances artificielles qui sont par ailleurs plus précoces à Amance $(2 \mathrm{j})$ et à Gennes (5 j) que les provenances naturelles; elle est faible pour le groupe 1. Pour les provenances naturelles, l'héritabilité de la vigueur semble assez forte comparativement aux observations effectuées par Légnaté (1986) sur la partie polonaise de l'aire naturelle ( $h^{2}=0,19-0,23$ à 13 ans).

Les corrélations génétiques entre hauteur et angle (tableau VIII) sont favorables mais significatives seulement dans 1 cas sur 4. Entre le débourrement et l'angle, elles sont, soit favorables mais non significatives pour les provenances artificielles, soit défavorables et significatives à Gennes (GR1). Les corrélations hauteurdébourrement ne sont pas significativement différentes de 0 .

Une des possibilités de valorisation de ce matériel roumain est la création d'un verger à graines de clones cinstitué à partir du groupe GRT qui rassemble le plus grand nombre de génotypes à Amance. Le choix des meilleurs clones a été réalisé par une sélection combinée individufamille pour le débourrement et la hauteur. L'angle moyen de branchaison étant satisfaisant $\left(67-70^{\circ}\right)$ et associé favorablement à $\mathrm{H} 88$, son coefficient de pondération dans

Tableau VII. Héritabilités au sens strict et intervalles de confiance au seuil de 95\%. GR1 (Gennes) comporte 33 familles, GRT (Amance) en a 96; le groupe des artificielles comporte 17 familles à Gennes et 19 a Amance.

GR1 ou GRT

\begin{tabular}{lllllll}
\hline \multirow{2}{*}{ Gennes } & $\mathrm{H}_{10}$ & GR1 & 0,32 & $(0,23-0,46)$ & 0,37 & $(0,24-0,67)$ \\
& DEB & GR1 & 0,24 & $(0,14-0,36)$ & 0,57 & $(0,37-1,09)$ \\
& ANG & GR1 & 0,30 & $(0,21-0,44)$ & 0,16 & $(0,08-0,34)$ \\
Amance & & & & & & \\
& $H_{10}$ & GRT & 0,39 & $(0,25-0,56)$ & 0,46 & $(0,26-0,91)$ \\
& DEB & GRT & 0,43 & $(0,29-0,61)$ & 0,78 & $(0,53-1,39)$ \\
& ANG & GRT & 0,35 & $(0,22-0,51)$ & 0,37 & $(0,21-0,74)$ \\
\hline
\end{tabular}


Tableau VIII. Corrélations génétiques additives. GR1 (Gennes) comporte 33 familles, GRT (Amance) en a 96; le groupe des artificielles comporte 17 familles à Gennes et 19 à Amance.

\begin{tabular}{lllrlll}
\hline \multirow{2}{*}{ Gennes } & $\mathrm{H}_{10} \times$ DEB & GR1 & $-0,13$ & $\mathrm{~ns}$ & 0,17 & $\mathrm{~ns}$ \\
& $\mathrm{H}_{10} \times$ ANG & GR1 & 0,68 & $* *$ & 0,16 & $\mathrm{~ns}$ \\
& DEB $\times$ ANG & GR1 & $-0,54$ & $*$ & 0,36 & $\mathrm{~ns}$ \\
Amance & $\mathrm{H}_{10} \times$ DEB & GRT & 0,19 & $\mathrm{~ns}$ & 0,24 & $\mathrm{~ns}$ \\
& $\mathrm{H}_{10} \times$ ANG & GRT & 0,22 & $\mathrm{~ns}$ & 0,55 & $\mathrm{~ns}$ \\
& DEB $\times$ ANG & GRT & 0,05 & ns & 0,46 & $\mathrm{~ns}$ \\
\hline
\end{tabular}

l'index a été fixé à 0 . La figure 5 montre qu'un poids croissant accordé au débourrement dans l'index entraîne une légère diminution de l'espérance de gain sur la hauteur; ceci est la conséquence de la très faible corrélation entre les 2 caractères ( $r=0,19 \mathrm{~ns}$ ). Le compromis de sélection a été choisi de telle sorte que la tardiveté obtenue soit sensiblement du même ordre que celle du témoin le plus tardif. Par rap-

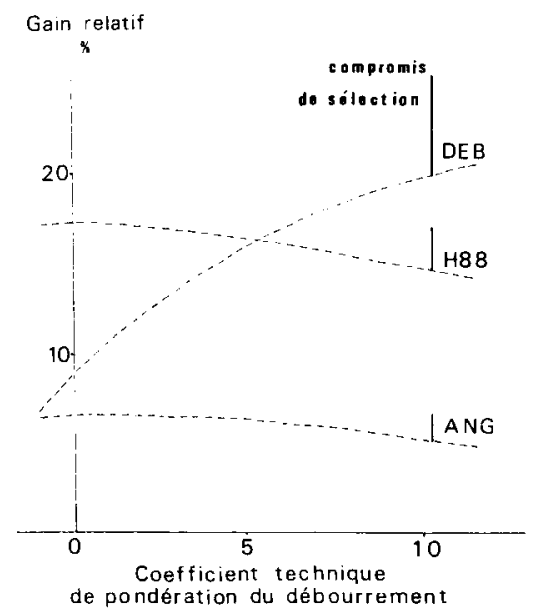

Fig 5. Simulation de sélection à Amance pour le groupe GRT. Les coefficients de pondération de la hauteur et de l'angle de branchaison sont respectivement 1 et 0 . L'écart entre le gain maximal et le gain au compromis est indique par une ligne verticale pour chaque caractère. port à la population initiale (GRT), la sélection des $10 \%$ meilleurs indices permet d'espérer une descendance du verger à la fois très vigoureuse $(+12 \%$, soit $72 \%$ du maximum) et aussi tardive que Chapois ( $+4 \mathrm{j}$, soit $73 \%$ du maximum) tout en ayant une bonne branchaison $1+5 \%$, soit $60 \%$ du maximum). Les 161 individus sélectionnés correspondent à 52 familles (sur les 96 initiales); aucune provenance n'a été exclue.

\section{DISCUSSION ET CONCLUSION}

Malgré les limites indiquées dans la section Matériel et Méthodes, cette étude apporte les premières informations sur les paramètres génétiques à 10 ans de quelques provenances roumaines d'épicéa commun.

Pour les caractères quantitatifs, la discrimination entre unités génétiques est meilleure pour le dispositif implanté à Gennes que pour celui d'Amance (tableau III). Ceci peut être dû à 2 «effets». D'une part, le dispositif expérimental de Gennes contrôle mieux l'effet milieu par des blocs plus petits (42 arbres contre 64) et permet une meilleure précision grâce à un plus grand nombre d'individus par provenance 
(219 contre 163) et par famille (38 contre 21). D'autre part, on peut penser qu'un site moins fertile comme Gennes (d'après sa hauteur moyenne) "éclaterait» mieux la variabilité; l'apparition de conditions limitantes variées révélant l'expression de nombreux gènes. Sur un site fertile (Amance), ces conditions n'apparaîtraient qu'après de nombreuses années, comme le suggère l'augmentation des effets provenance au cours du temps. Au contraire, la présence de fourches est associée à la vigueur (niveaux provenance et descendance) et ne se manifeste suffisamment que sur un site fertile de type Amance.

Ainsi, l'implantation d'un dispositif sur plusieurs sites contrastés peut aider à mieux révéler la variabilité globale du matériel. Toutefois, le choix de sites trop différents peut conduire à sortir des stations normales de reboisement en épicéa commun. Ainsi, le site d'Amance paraît plus fertile que ce qui est habituel pour cette essence.

Malgré la différence de fertilité entre les 2 sites, les interactions génotype $x$ environnement demeurent pratiquement inexistantes. Le matériel roumain apparaît particulièrement stable tant aux niveaux provenance et descendance, que pour les blocs, sites et années.

En tenant compte des limites de cette étude, une certaine concordance peut s'observer entre les résultats présentés ici et les informations disponibles, en particulier les données écologiques (Ministerul Silviculturii, 1988). Ainsi, les 2 provenances artificielles se distinguent des autres, en particulier de celles du Bihor, tant par leur précocité (fig 4) que par les tests de Kullback. De même, les provenances du Bihor diffèrent de celles de la zone centre par la vigueur. Enfin, la provenance GURG proche de la Bucovine, mais n'appartenant pas à la même zone écolo- gique s'en distingue par une faible vigueur à Amance.

En revanche, aucune distinction n'apparaît entre la provenance NEAS située sur la face méridionale de l'arc carpatique et les provenances SINM et COMA proches du "pôle du froid" (fig 3). De même, la forte variabilité des provenances de Bucovine pour la hauteur à Gennes ne semble pas associée aux données écologiques et pourrait résulter, soit d'une faiblesse de l'échantillonnage, soit d'un éclatement de la variabilité comme cela a été observé sur des provenances polonaises (Légnaté, 1986).

Pour certaines provenances, aucune variabilité interfamiliale n'a pu être mise en évidence, à la fois sur les 2 sites et pour les 3 caractères quantitatifs. Cela peut résulter d'un biais dans l'échantillonnage (effectif, représentativité). Pour les autres provenances, les héritabilités sont élevées, notamment pour la vigueur. Les corrélations génétiques sont plutôt défavorables pour le groupe de descendances étudiées à Gennes, et favorables à Amance sur l'ensemble du matériel (GRT). Sur ce site, les simulations de sélection permettent d'espérer des gains génétiques importants (tableau IX).

La valorisation de ces résultats, et principalement ceux obtenus à Amance, peut se faire par l'importation de graines ou la constitution de vergers à graines. L'utilisation des provenances CIMP et MOLD conduirait à un gain de $27 \%$ sur la vigueur par rapport à Gérardmer, pour un débourrement tardif et un angle de branchaison élevé (tableau IX). Ces peuplements portegraines ont été cartographiés en Roumanie par Enescu (1984); un approvisionnement en graines roumaines est donc envisageable.

D'autre part, pour une orientation "Verger à Graines", la sélection d'une cin- 
Tableau IX. Comparison des sorties variétales envisagées.

\begin{tabular}{lccc}
\hline & $H_{10}(\mathrm{~cm})$ & DEB $(j)$ & ANG (\%) \\
\hline Moyennes de Chapois & 311 & 23,5 & 63,7 \\
\multicolumn{1}{c}{ Gérardmer } & 321 & 13,8 & 64,2 \\
Moyenne des 12 provenances (GRT) & 375 & 19,9 & 70,7 \\
Gain en \% (") & $17 \%$ & & $10 \%$ \\
Moyenne de CIMP et MOLD & 407 & 20,5 & 72,5 \\
Gain en \% (") & $27 \%$ & & $13 \%$ \\
Valeur espérée de la descendance & & & \\
d'un verger à graines (50 indiv) & 430 & 24,0 & 75,0 \\
Espérance de gain (") & $34 \%$ & & $17 \%$ \\
\hline
\end{tabular}

(") Les gains sont estimés par rapport au meilleur témoin.

quantaine de clones non apparentés fournirait un gain de $34 \%$ par rapport à Gérardmer, soit $5 \%$ de plus que les meilleures provenances roumaines. Cette opération conduirait à une certaine indépendance de l'approvisionnement en graines de qualité. Toutefois, elle nécessiterait des investissements suffisamment lourds pour justifier une certaine prudence. Ainsi, les résultats devront être confirmés à un âge plus avancé. En particulier, il sera nécessaire d'analyser d'autres caractères comme la qualité mécanique du bois. Par ailleurs, un échantillonnage plus complet pourrait être réalisé sur les meilleures populations afin d'obtenir des informations plus fiables sur leur variabilité naturelle.

Les résultats présentés ici mettent dès maintenant en valeur les bonnes performances de certaines provenances et descendance roumaines. L'utilisation de ce matériel peut procurer une amélioration certaine de l'espèce par voie générative. La confirmation de ces résultats permettra de juger de la place que devra occuper le matériel roumain dans notre stratégie d'amélioration de l'épicéa commun.

\section{REFERENCES}

Bachacou J, Masson JP, Millier C (1981) Manuel de la programmatheque statistique AMANCE 81, INRA, $516 \mathrm{p}$

Baradat $P$ (1984) Un modèle généralisé pour l'étude de l'interaction génotype-milieu. INRA, Laboratoire d'amélioration des arbres forestiers, Pierroton

Becker WA (1984) Manual of quantitative genetics. Academic enterprises Pullman, Washington, $190 \mathrm{p}$

Christophe C (1979) Stratégies de sélection sur indices à partir de plusieurs populations. Ann Amélior Plant 29, 227-246

Enescu V (1984) Réalisation et perspectives des recherches génétiques forestières en République Socialiste de Roumanie. Rev For Fr XXXVI, 5, 425-432

Ferrand J Ch (1986) Les programmes d'amélioration. Résultats actuels et escomptés pour l'épicéa commun. Rev For Fr, 125s-128s

Héois B (1989) Étude de la structure génétique de populations roumaines d'épicéa commun, corrélations génétiques, héritabilités, espérances de gain génétique. DEA, INAPG, Rapport INRA Orléans, $51 \mathrm{p}$

Legendre L, Legendre P (1984) Ecologie numérique, tome $2,2^{\circ}$ edn. Masson, Paris, 29 et $182-183$ 
Légnaté NH (1986) Étude de la structure génétique des populations polonaises d'épicéa commun (Picea abies Karst). Corrélations génétiques. Héritabilités. Espérances de gains génétiques. DEA, Université de Paris Sud, Rapport INRA Orléans, $42 p$

Ministerul Silviculturil (1988) Zonele de recoltare a semintelor forestiere in RS România, Bucuresti, $57 \mathrm{p}$

Portefaix C (1987) Exploration de la variabilité génétique du Pin laricio de Corse, Pinus nigra Arn ssp; Laricio var Corsicana Loud Perspectives pour l'amélioration de l'espèce. Thèse de docteur ingénieur, INAPG, $105 \mathrm{p}$

Schmidt-Vogt H (1977) Die Fichte. Band 1, Taxonomieverbreitung. Morphologie. Okologie. Waldgesellschaften. Hamburg and Berlin, Paul Parey, $647 p$

Wricke G (1962) Uber eine Methode zur Erfassung der ökologischen Streubreite in Feldversuchen. Z Pflanzenschutz 47, 92-96 